\section{Immediate vs delayed shock on a spatial dimension*t;}

\author{
W. H. TEDFORD, JR. \\ Southern Methodist University, Dallas, Tex. 75222 \\ and \\ PATRICIA K. LEIBENSPARGER \\ Muskingum College, New Concord, Ohio 43762
}

Comparisons were made among preferences for short, medium, or long spatial delays of shock, using 36 female albino rats. Significant preferences were found for long delay over either short or medium but for short over medium. This was interpreted in terms of the ambiguity of the choice situation: when punishment is absolutely certain, Ss prefer to have it immediately; when there is some ambiguity present, delayed punishment is selected.

When $\mathrm{S}$ is given a choice between an immediate and a delayed shock, the question of which he will choose is important for several theoretical formulations concerning punishment (Miller, 1959; Mowrer, 1950); yet the question has not been settled empirically. Knapp, Kause, \& Perkins (1959, Experiment I) found that rats chose immediate shock over a $30-\mathrm{sec}$ delayed shock. Hare (1966) and Cook $\&$ Barnes (1964) observed much the same performance in humans. On the other hand, Butler's (1959) results indicated that rats preferred shocks delayed by $2,4,8$, or $16 \mathrm{sec}$ to those delivered immediately after the response. Renner \& Specht (1967, Group 1) supported this finding. The less aversive nature of delayed shock, and hence, a preference for immediate shock, could be inferred from studies demonstrating faster learning with immediate than with delayed shock; such results have been found with both rats (Tedford, 1969) and humans (Banks \& Vogel-Sprott, 1965). Renner \& Houlihan (1969) apparently feel that escape from the area where shock is administered is the key variable; their Ss preferred immediate shock under escape conditions but delayed shock under no-escape conditions.

The present experiment was designed to investigate this problem on a spatial dimension. It was hoped that adding movement cues during the passage of time would make the intervals more discriminable. This should make the choice more clearly defined for $\mathbf{S}$ and perhaps lead to more consistent results.

A Y-maze was constructed in which $S$ could be shocked as soon after the

*This experiment was performed at Oberlin College and was supported by NSF Grant No. GY-4241 (URP).

TThe authors wish to thank Dr. C. C. Perkins, Jr., for suggestions on the experimental design and Mr. Steve Fleishman for assistance in collecting pilot data. choice as feasible. That is, "immediate shock" occurred "one rat length" beyond the choice point, so that a door could be lowered behind $S$ to prevent him from jumping back past the choice point at shock onset. Two other distances, corresponding to medium delay and long delay, were also selected for shock administration. METHOD

Data are reported from 36 female albino rats of the Sprague-Dawley strain. One $S$ died during the experiment and was replaced. Weights were maintained at $85 \%$ of ad lib weight during the experiment.

A scale drawing of the apparatus is shown in Fig. 1. Flooring consisted of two $3.7-\mathrm{cm}$ strips of steel separated by a 1.2-cm gap. Guillotine doors were raised and lowered manually. Shock was initiated by breaking a photocell beam; a relay insured that only one shock could be delivered on each trial. A $9-V$ dc shock lasting .5 sec was delivered through the floor by a constant-voltage source. The three photocells on each side were designated as short (S), medium (M), and long (L).

All Ss were given $15 \mathrm{~min}$ of exploration in each arm of the maze, with food present. Starting on the next day, they were run for six trials per day on a schedule of one free choice followed by a forced choice to the opposite side. This continued until they had made eight consecutive trials in less than $30 \mathrm{sec}$ each. Reward consisted of three 45-mg Noyes pellets on each trial.

On the day following criterion, shock was introduced. Ss were assigned randomly to one of six groups: $L$ vs $S, L$ vs $M$, and $M$ vs $S$, with longer delay counterbalanced to occur on the right for half of the groups. Four trials per day were run for 10 days: on the first 5 days, the second and fourth trials were forced to counterbalance the first and third; on the last 5 days, four free choices were allowed. Thus, each $\mathbf{S}$ made a total of 30 free choices.

\section{RESULTS}

The major finding was that $\mathrm{L}$ was selected over $M(t=3.24, d f=11$, $p<.01)$ and $S(t=4.63, d f=11$, $\mathrm{p}<.001$ ) but that $\mathrm{S}$ was chosen more often than $M(t=2.22, \quad d f=11$, $p<.05$ ). The same significance levels were obtained in an analysis of variance, which also indicated that there was no significant change in performance over trials.

Figure 2 indicates the percent choice of the longer delay on each day of testing. Points for the last 5 days are based on twice as many choices as those for the first 5 days; plotting by single trials or blocks of trials fails to indicate any trend not shown in Fig. 2. The experiment was designed to terminate after 10 days because it was felt that shock adaptation would occur and that choice would return to near the $50 \%$ level. However, percent choice remained fairly constant over the last 20 trials. There was an observable trend for many Ss to decrease in running speed near the end of the experiment.

The right arm of the maze was chosen slightly more often than the

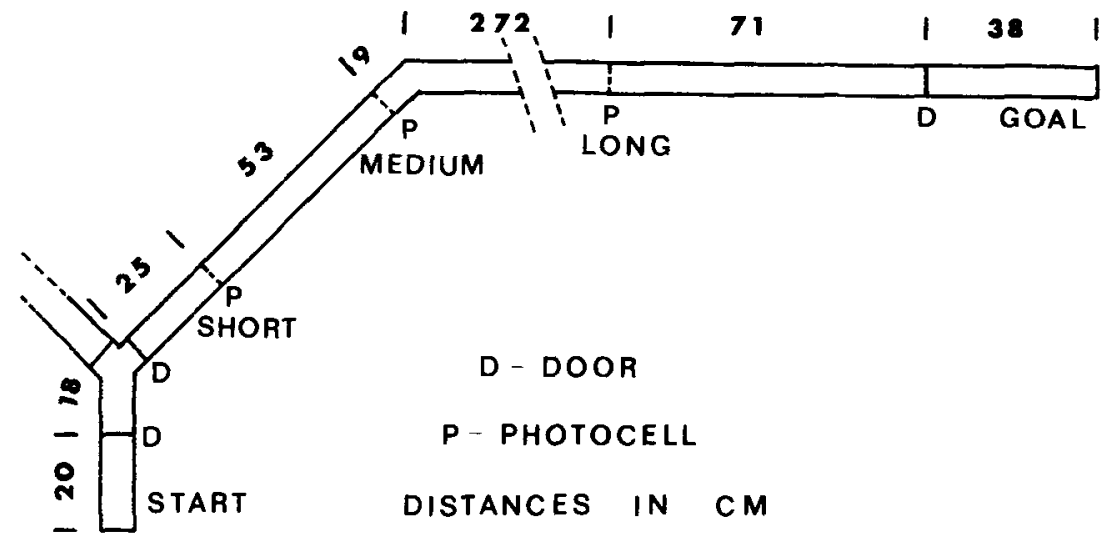

Fig. 1. Scale drawing of maze. 


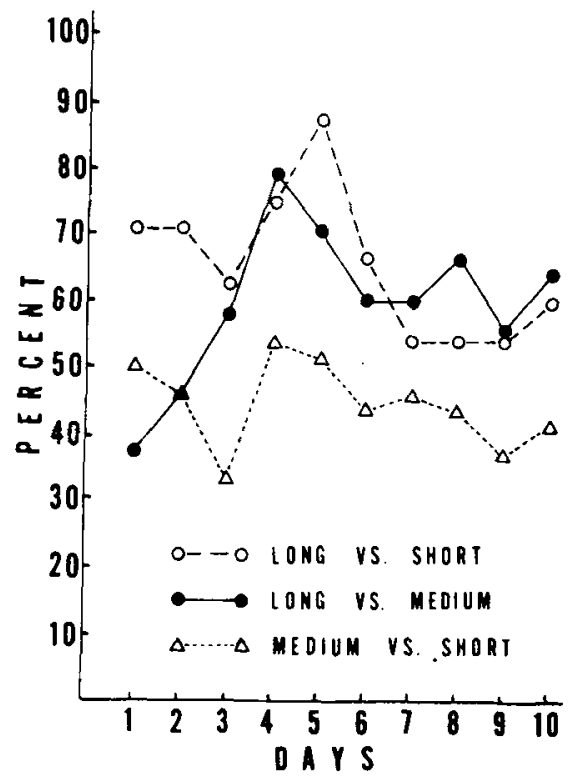

Fig. 2. Choice of longer delay on each test day.

left, but not significantly so: $t=.14$, df $=\mathbf{3 5}$.

\section{DISCUSSION}

Although these results may at first seem contradictory, it is felt that they fit a pattern which may be observed in the literature. It should be noted that the photocell beams for both $S$ and $M$ can be seen from the choice point. In this case, it is obvious that shock will occur and immediate shock is selected. This corresponds to Hare's Ss, who were told that shock would occur, and to the Knapp et al Ss, who were forced to choose between black and white goalboxes which were both associated with shock. In contrast, $S s$ in the present study in the $\mathrm{L}$ vs $\mathrm{S}$ or $\mathrm{L}$ vs $M$ groups were presented with "out of sight" vs "visible" shock points. The out of sight point being more similar to no shock, Ss selected it. There is a similarity here to Butler's Ss. Although he placed each $\mathbf{S}$ between doors with vertical or horizontal stripes, the doors were removed prior to $\mathbf{S}$ actually making a choice. At the "moment of truth," $S$ found himself in a uniform box with both ends similar to the point on which he stood and at which he had never received shock. Again, delayed shock being more similar to no shock, he chose the delay.

The principle being put forth is a simple one. As long as $\mathrm{S}$ is certain that he will receive shock, he prefers to have it immediately rather than after some delay. This is in accord with Mowrer's (1950) idea of "dread." However, if there is enough ambiguity in the situation to allow even a remote possibility that shock will not occur, $S$ selects the delayed-shock alternative. This may be because of the greater similarity of delayed shock to no shock, or it may be simply a desire to postpone unpleasant consequences if there is some chance that they may be avoided altogether.

It is felt that the ambiguity in the "Renner box" comes from the similarity of the startbox and the delay boxes. Since $S$ is never shocked in the startbox, and yet the startbox suddenly becomes part of one delay chamber or the other, this analysis would predict that $S$ would choose delayed shock. When cues of the type employed by Renner \& Houlihan (1969) are introduced, i.e., the delayed shock is delivered in a compartment painted similarly to the startbox but the immediate shock occurs in a differently painted compartment, the choice of delayed shock should increase. This is, in fact, what occurred in their experiment (Figs. 1 and 3). The addition of an escape condition, whereby $S$ can move into a differently painted goalbox, alters the situation. There is no ambiguity about the safety of the goalbox; $S$ desires to reach it as quickly as possible and chooses immediate shock. Again, the effect of cues should increase the percent choice, as it does in their Fig. 1. In describing this figure, they state, "... the presence of cues did not influence the selection between the immediate or delayed side." While this is true for the overall cue effect, inspection of the figure indicates an interaction between cue/no cue and escape/no escape. Failure of cues to increase preference for immediate shock under escape conditions in another case (their Fig. 3) may be due to the bias for delay introduced by having the delayed-shock compartment painted the same as the startbox.

It is felt that this principle is in accordance with all results mentioned above except one. Hare (1966) also ran a group of nine Ss who received shock only $50 \%$ of the time, and they also preferred immediate shock. The present authors would predict a preference for delayed shock due to the uncertainty of punishment. However, it appears that the Ss were verbally instructed to expect shock $100 \%$ of the time, and there is a trend away from the immediate-shock preference. Hare's Ss received 30 trials, and it is felt that, had his experiment continued, this trend would have developed into a delayed-shock preference.

The present authors would not disagree with Renner and Houlihan on the importance of specific conditions in this type of experiment. However, they do disagree strongly with the statement that the factors determining relative aversiveness of delayed or immediate shock may be unimportant because the preferences are transient and temporary. The gradual return of choices to the $50 \%$, or no-preference, level is more likely to be due to shock adaptation than to feelings of "hopelessness." If the latter were a correct interpretation, human Ss who were instructed that shock occurred on every trial would show no preference from the start. This does not seem to be the case. Delivery of delayed vs immediate punishment is a choice faced by parents, teachers, etc., so frequently that the factors determining relative aversiveness are extremely important for both applied and theoretical psychology.

\section{REFERENCES}

BANKS, R. K., \& VOGEL-SPROTT, M. Effect of delayed punishment on an immediately rewarded response in humans. Journal of Experimental Psychology, 1965, 70, 357-359.

BUTLER, D. Two-choice behavior as a function of the delay between response and punishment. Unpublished PhD dissertation, Northwestern University, 1958.

COOK, J. O.. \& BARNES, L. W. Choice of delay of inevitable shock. Journal of Abnormal \& Social Psychology, 1964, 68, 669-672.

HARE, R. D. Preference for delay of shock as a function of its intensity and probability. Psychonomic Science, 1966. 5. 393-394.

KNAPP, R. K., KAUSE, R. H., \& PERKINS, C. C. Immediate vs. delayed shock in T-maze performance. Journal of Experimental Psychology, 1959, 58, 357-362.

MILLER, N. E. Liberalization of basic $S-R$ concepts: Extensions to conflict behavior, motivation, and social learning. in S. Koch (Ed.). Psychology: A study of $a$ science. Vol. 2 . New York: McGraw-Hill, 1959. Pp. 196-292.

MOWRER, O. H. Learning theory and personality dynamics. New York: Ronald Press, 1950

RENNER, K. E., \& HOULIHAN, J. Conditions affecting the relative aversiveness of immediate and delayed punishment. Journal of Experimental Psychology, 1969, 81, 411-420.

RENNER, K. E., \& SPECHT, L. The relative utility or aversiveness of immediate and delayed shock and food. Journal of Experimental Psychology, 1967, 75, 568-579.

TEDFORD, W. H., JR. Effect of delayed punishment upon choice behavior in the white rat. Journal of Comparative \& Physiological Psychology, 1969, 69 673-676. 